\title{
The Puzzle of Generalized Osteoarthritis (OA) — Is OA a Systemic Enthesopathy?
}

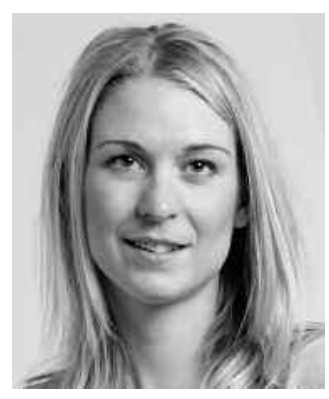

Osteoarthritis (OA) is a common condition that has a major influence on patients' health-related quality of life. Unfortunately, the riddle concerning the pathogenesis of the disease is still not solved. Generally, we believe that OA is a failure of the whole joint caused by a combination of systemic risk factors and local biomechanical factors ${ }^{1,2}$.

Hand $\mathrm{OA}$ is frequent in persons with $\mathrm{OA}$ in the larger joints, and may represent a marker of a generalized susceptibility to OA. Kellgren and Moore suggested the concept of generalized $\mathrm{OA}$ in the early $1950 \mathrm{~s}^{3}$, and numerous later studies have confirmed an association between hand and knee $\mathrm{OA}$ and to a lesser extent hip and spine $\mathrm{OA}^{4,5,6}$. Recently, the association was confirmed with modern imaging techniques demonstrating an association between radiographic joint space narrowing in the finger joints and reduced knee cartilage thickness, and between osteophytes in the finger joints and radiographic knee $\mathrm{OA}^{7}$. Although there is ample evidence of an association between hand and knee OA, there is still no consensus about the definition of generalized $\mathrm{OA}^{8}$. The underlying mechanisms beyond this association are not fully understood, although genetic, hormonal, nutritional, neurological, and autoimmune mechanisms have been suggested. Further, one cannot rule out that the observed association is due to common environmental risk factors for hand and knee OA. In this issue of The Journal, Gibson, et al explore the concept of generalized $\mathrm{OA}$ and ask whether the disease is related to a systemic enthesopathy ${ }^{9}$.

Entheses are defined as the insertion sites of tendons, ligaments, fascia, or the articular capsule into bone ${ }^{10}$. The term enthesopathy refers to any pathological involvement of the entheses, including inflammatory, metabolic, traumatic, or degenerative processes. Hence, enthesopathy is a common feature in many musculoskeletal disorders, including both systemic diseases such as spondyloarthropathies and local processes related to injuries or overuse ${ }^{10}$.

Enthesopathic changes are demonstrated in both hand OA and knee OA. Tan, et al performed high-resolution mag- netic resonance imaging of the finger joints and found high frequency of collateral ligament disruption, thickening, and inflammation in joints with established OA, but abnormalities were also found in joints where the cartilage was mostly preserved $^{11}$. Osteophytes, erosions, and bone marrow lesions (BML) were seen in close anatomic relationship to the ligaments, and the authors suggested that the ligaments might play a role in the pathogenesis of hand OA. Similarly, Hernandez-Molina, et al showed that partial/complete tear of the anterior cruciate ligament of the knee was cross-sectionally associated with central BML ${ }^{12}$. However, no previous study has shown whether these changes are local events related to biomechanical and tissue stresses or are the result of a systemic process. The current study by Gibson, et al is the first to examine the possible association between enthesopathic changes in the hand and knee, and is therefore an important contribution to the puzzle of generalized $\mathrm{OA}^{9}$.

Gibson, et al have performed a matched case-control study comparing the frequency of hand enthesophytes in participants with and without central BML in the knees 9 . The participants were recruited from the Framingham Offspring and Community Cohorts, and were not selected based on the presence of knee symptoms or radiographic OA. Central BML were by definition located in the area of the cruciate ligament attachment, and could therefore serve as markers for knee enthesopathy. These BML are different from other BML in knee OA, being subcortical and related to weight-bearing. Gibson, et al concluded that participants with central BML in the knee were not more likely to have radiographic enthesophytes in the finger joints, and the same results were shown in analyses restricted to participants with radiographic knee OA.

The first topic of discussion is whether the observed enthesopathic changes are related to a systemic process or to the local biomechanical environment. The results from the current study do not support OA as a systemic enthesopathy ${ }^{9}$, and the enthesopathic changes seemed to be more related to local "wear and tear" and biomechanical stresses.

See Relation of hand enthesophytes with knee enthesopathy, page 359

Personal non-commercial use only. The Journal of Rheumatology Copyright $@$ (2012. All rights reserved. 
Even though the finger joints are not "weight-bearing" (in contrast to the knees), they are definitely "load-bearing"13. Most of the intraarticular forces in the finger joints are caused by contraction of muscles that span the joints. Bone spurs can develop at the insertion sites of tendons as a response to this stress. Tendons may also fan out their attachment sites as an adaptive mechanism in order to distribute the force over a greater area, leading to development of enthesophytes along the midshafts ${ }^{14}$. Similarly, central BML in the knee may represent local bone remodeling caused by changes in the biomechanical environment related to tears of the cruciate ligaments.

Is the existence of a systemic enthesopathy possible, but obscure in this study for methodological reasons? One could possibly argue that hand enthesophytes are not sensitive and specific measures of systemic enthesopathy. Enthesophytes are developed by endochondral ossification ${ }^{14}$, and these bone changes may represent late features. Gibson, et al assessed only midshaft enthesophytes on radiographs with a posteroanterior view. Enthesophytes at the insertion of the extensor tendons, which are most easily seen by oblique or lateral views, were therefore not assessed. Central BML may be more sensitive markers of enthesopathy than bone proliferation, but their natural course is unknown and they may possibly fluctuate in size and presence. It is also possible that enthesopathic changes related to systemic enthesopathies and local biomechanical processes (i.e., wear and tear or trauma) coexist, although the latter may be more dominant (at least in this study). Trauma is especially relevant for the knees, but the frequency of recalled knee injury in those with central BML was not provided in the current report. Based on these considerations, we cannot rule out the existence of a systemic component behind the enthesopathic changes, in addition to the apparently stronger local biomechanical component.

Enthesopathy is associated with a variety of metabolic and endocrine disorders in addition to rheumatic disorders and drug-induced tendinopathies ${ }^{10}$. Diabetes mellitus and chondrocalcinosis have also been linked to $\mathrm{OA}^{15,16}$, and systemic enthesopathic changes could possibly represent the link between these disorders. The association between diabetes and enthesophytes was also confirmed in the current study, with a particularly strong association in those with no central $\mathrm{BML}^{9}$. Chondrocalcinosis is seen as linear cartilage calcifications on the knee radiographs, but also frequently involves the entheses. Due to the high prevalence in the middle-aged and elderly, this may be the most significant single cause of systemic enthesopathy. Chondrocalcinosis and OA commonly co-occur, but the association seems not to be related only to age ${ }^{16}$. Unexpectedly, longitudinal studies have not shown increased risk of cartilage loss associated with chondrocalcinosis ${ }^{17}$.

The second discussion point is whether enthesopathic changes - the result of either local or systemic processes
- are related to $\mathrm{OA}$, or are merely co-occurring phenomena. A previous study of skeletons demonstrated strong associations between bony features of $\mathrm{OA}$ and generalized enthesophyte formation ${ }^{18}$. The age of the skeleton could not be exactly determined, and the association could possibly be confounded by age. Indeed, in hand OA the association between radiographic enthesophytes and osteophytes seemed to be mainly age-related ${ }^{19}$. Tan, et al found ligament abnormalities in unaffected joints in patients with hand OA, suggesting that ligament abnormalities could represent early features of $\mathrm{OA}^{11}$. However, the same abnormalities were also frequent in elderly, healthy controls, and the predictive validity of these lesions remains unknown due to the crosssectional study design. The co-occurrence of osteophytes and enthesophytes may also be due to common biomechanical risk factors. As noted, the loading of finger joints is mostly due to contraction of muscles that span the joints ${ }^{13}$. Bone spurs can develop at the sites of attachment of tendons due to mechanical stresses, and the force generated on the joint surface area may simultaneously have a detrimental effect on the cartilage. In knee OA, it is well known that ligament degeneration, instability, and tears are risk factors for knee OA development due to an altered biomechanical joint environment ${ }^{20}$. The central BML could also arise from traumatic tension of the insertion of cruciate ligaments, triggered by laxity in OA joints. Hence, whether enthesopathic changes are part of the OA syndrome or only a co-occuring phenomenon due to common risk factors remains a subject of debate.

In conclusion, Gibson, et al found a high frequency of enthesophytes in the hands and central BML in the knees 9 . Interestingly, there was no association between the enthesopathic changes at the 2 locations, suggesting that the changes were mainly biomechanically induced. Hence, a systemic enthesopathy seems not to be an important component of the puzzle of generalized OA.

\author{
IDA K. HAUGEN, MD, \\ Department of Rheumatology, \\ Diakonhjemmet Hospital, \\ PO Box 23 Vinderen, \\ 0319 Oslo, Norway
}

Address correspondence to Dr.Haugen.E-mail: ida.haugen@diakonsyk.no

Supported by grants from the South-Eastern Norway Regional Health Authority.

\section{ACKNOWLEDGMENT}

I would like to thank Tore K. Kvien (Diakonhjemmet Hospital, Oslo) and Martin Englund (Lund University, Sweden) for valuable input.

\section{REFERENCES}

1. Hunter DJ. Insights from imaging on the epidemiology and pathophysiology of osteoarthritis. Radiol Clin North Am 2009;47:539-51. 
2. Brandt KD, Radin EL, Dieppe PA, van de Putte L. Yet more evidence that osteoarthritis is not a cartilage disease. Ann Rheum Dis 2006;65:1261-4.

3. Kellgren J, Moore R. Generalized osteoarthritis and Heberden's nodes. Br Med J 1952;1:181-7.

4. Hirsch R, Lethbridge-Cejku M, Scott WW, Reichle R, Plato CC, Tobin J, et al. Association of hand and knee osteoarthritis: evidence for a polyarticular disease subset. Ann Rheum Dis 1996;55:25-9.

5. Dahaghin S, Bierma-Zeinstra SMA, Reijman M, Pols HAP, Hazes JMW, Koes BW. Does hand osteoarthritis predict future hip or knee osteoarthritis? Arthritis Rheum 2005;52:3520-7.

6. Bijkerk C, Houwing-Duistermaat JJ, Valkenburg HA, Meulenbelt I, Hofman A, Breedveld FC, et al. Heritabilities of radiologic osteoarthritis in peripheral joints and of disc degeneration of the spine. Arthritis Rheum 1999;42:1729-35.

7. Haugen IK, Cotofana S, Englund M, Kvien TK, Dreher D, Nevitt $\mathrm{M}$, et al. Hand joint space narrowing and osteophytes are associated with MRI-defined knee cartilage thickness and radiograpic knee osteoarthrtitis - Data from the Osteoarthritis Initiative (OAI). J Rheumatol 2012;39:161-6.

8. Dougados M, Nakache JP, Gueguen A. Criteria for generalized and focal osteoarthritis. Rev Rhum Engl Ed 1996;63:569-75.

9. Gibson N, Guermazi A, Clancy M, Grayson P, Aliabadi P, Roemer F, et al. Relation of hand enthesophytes with knee enthesopathy: Is osteoarthritis related to a systemic enthesopathy? J Rheumatol 2012;39:359-64.

10. Slobodin G, Rozenbaum M, Boulman N, Rosner I. Varied presentations of enthesopathy. Semin Arthritis Rheum 2007;37:119-26

11. Tan AL, Grainger AJ, Tanner SF, Shelley DM, Pease C, Emery P, et al. High-resolution magnetic resonance imaging for the assessment of hand osteoarthritis. Arthritis Rheum 2005; 52:2355-65.
12. Hernández-Molina G, Guermazi A, Niu J, Gale D, Goggins J, Amin S, et al. Central bone marrow lesions in symptomatic knee osteoarthritis and their relationship to anterior cruciate ligament tears and cartilage loss. Arthritis Rheum 2008;58:130-6.

13. Radin EL, Parker HG, Paul IL. Pattern of degenerative arthritis. Preferential involvement of distal finger-joints. Lancet 1971;1:377-9.

14. Benjamin M, Rufai A, Ralphs JR. The mechanism of formation of bony spurs (enthesophytes) in the achilles tendon. Arthritis Rheum 2000;43:576-83.

15. Berenbaum F. Diabetes-induced osteoarthritis: From a new paradigm to a new phenotype. Ann Rheum Dis 2011;70:1354-6.

16. Felson DT, Anderson JJ, Naimark A, Kannel W, Meenan RF. The prevalence of chondrocalcinosis in the elderly and its association with knee osteoarthritis: The Framingham Study. J Rheumatol 1989; $16: 1241-5$

17. Neogi T, Nevitt M, Niu J, LaValley MP, Hunter DJ, Terkeltaub R, et al. Lack of association between chondrocalcinosis and increased risk of cartilage loss in knees with osteoarthritis: Results of two prospective longitudinal magnetic resonance imaging studies. Arthritis Rheum 2006;54:1822-8

18. Rogers J, Shepstone L, Dieppe P. Is osteoarthritis a systemic disorder of bone? Arthritis Rheum 2004;50:452-7.

19. Kalichman L, Malkin I, Kobyliansky E. Hand bone midshaft enthesophytes: The influence of age, sex, and heritability. Osteoarthritis Cartilage 2007;15:1113-9.

20. Øiestad BE, Engebretsen L, Storheim K, Risberg MA. Knee osteoarthritis after anterior cruciate ligament injury: A systematic review. Am J Sports Med 2009;37:1434-43.

J Rheumatol 2012;39:203-5; doi:10.3899/jrheum.111151 